\title{
Measuring and Managing Blood Pressure in a Primary Care Setting: A Pragmatic Implementation Study
}

\author{
John Doane, MD, Jenni Buu, PharmD, M. Jason Penrod, MD, Michelle Bischoff, MD, \\ Molly B. Conroy, MD, MPH, and Barry Stults, MD
}

Background: Accurate blood pressure (BP) measurement is essential to hypertension diagnosis and management. Automated office blood pressure (AOBP) and home blood pressure measurement (HBPM) may improve assessment, but barriers exist in primary care settings.

Methods: We implemented an AOBP/HBPM program in a primary care clinic in 2015 to 2016. Patients with elevated BP determined by guideline-quality observed BP measurement and/or AOBP entered the HBPM program. Patients with average home BP $\geq 135 / 85 \mathrm{~mm} \mathrm{Hg}$ provided HBPM results for medication adjustment. Clinic staff and patients completed satisfaction questionnaires.

Results: Initial HBPM results in 183 patients with elevated office BP revealed white-coat BP elevation in 35\% of untreated patients and in 37\% of treated patients. The prevalence of white-coat BP elevation was similar whether enrollment BP was by observed BP or AOBP. Subsequent HBPM facilitated BP control in 49\% of patients with elevated home BP. Most providers, staff, and patients endorsed the utility of the program. Barriers to implementation included a temporary period of incorrect AOBP technique, patients failing to provide HBPM results, and incorrect HBPM technique.

Discussion: Our clinic-based AOBP/HBPM program detected white-coat BP elevation in one third of enrolled patients, facilitated control of home BP, and was acceptable to staff and patients. We identified barriers to be addressed to ensure sustainability. (J Am Board Fam Med 2018;31:375-388.)

Keywords: Blood Pressure, Hypertension, Implementation Science, Personal Satisfaction, Primary Health Care, Surveys and Questionnaires

Accurate blood pressure (BP) measurement is essential to effective hypertension diagnosis and management. Unfortunately, routine office blood pressure measurement $(\mathrm{OBP})$ is frequently inac-

This article was externally peer reviewed.

Submitted 27 November 2017; revised 9 February 2018; accepted 15 February 2018.

From the Department of General Internal Medicine, School of Medicine, University of Utah, Salt Lake City, UT (JD, MJP, MB, MBC, BS); Pharmacy Primary Care Services, University of Utah Health, Salt Lake City (JB).

Funding: This publication was in part supported by the Cooperative Agreement, 3U58DP004835, funded by the Centers for Disease Control and Prevention. Its contents are solely the responsibility of the authors and do not necessarily represent the official views of the Centers for Disease Control and Prevention or the Department of Health and $\mathrm{Hu}-$ man Services.

Conflict of interest: none declared.

Corresponding author: Molly B. Conroy, MD, MPH, School of Medicine, University of Utah, 30 N. 1900 E. Room 5R218, Salt Lake City, UT (E-mail: molly.conroy@hsc.utah.edu). curate due to incorrect measurement technique ${ }^{1-3}$ and/or "white-coat" BP elevation., 4 These 2 factors result in frequent overestimation of usual patient $\mathrm{BP}$ and consequent misdiagnosis and overtreatment of hypertension in $20 \%$ to $65 \%$ of persons with elevated office BP. ${ }^{5}$ Current US and international guidelines emphasize the necessity of accurate BP measurement in and out of the office, and this necessity will be magnified if the lower BP goals recommended by some guidelines are implemented. ${ }^{6-8}$ Most current guidelines recommend OBPM to screen for hypertension with confirmation of the diagnosis by out-of-office BP monitoring. ${ }^{6-11}$ A 24-hour ambulatory blood pressure monitoring study (ABPM) is preferred to confirm the diagnosis of hypertension. ${ }^{6-11}$ If $A B P M$ is unavailable or refused by the patient, home BP monitoring performed by the patient twice daily for 7 days is an acceptable alternative. ${ }^{6-11}$ 
However, ABPM is infrequently used in the United States ${ }^{12}$ due to limited availability ${ }^{13}$ and reimbursement ${ }^{14,15}$ and clinician unfamiliarity with its utility. ${ }^{14}$ In contrast, home blood pressure measurement (HBPM) is currently used by about 50\% of US hypertensive adults ${ }^{16}$ and $34 \%$ to $70 \%$ of US primary care clinicians. ${ }^{17}$ HBPM combined with 1 or more cointerventions (eg, clinician-guided medication titration, pharmacist/nurse comanagement with or without telemonitoring or digital health platforms, or algorithm-guided patient self-titration of medication) lowers BP and improves hypertension control rates in research studies. ${ }^{18-22} \mathrm{Im}^{-}$ portant barriers to effective implementation of HBPM into general US primary care practice include the considerable expense of interventions such as telemonitoring systems or adding staff for comanagement, the need for clinic redesign to incorporate HBPM training into a busy clinic setting, and concern about the accuracy of HBPM performed by patients in nonresearch settings. ${ }^{14,23-29}$

Automated office blood pressure measurement (AOBP), the performance and averaging of 3 to 5 sequential automated BP measurements by a validated device while patients rest alone ${ }^{30}$, may reduce white-coat BP elevation and decrease the need for subsequent out-of-office BP measurement. ${ }^{31,32}$ In patients with initially elevated office BP, AOBP is more time efficient than performing 3 guidelinerecommended observed office BP measurements. AOBP requires 4 to 7 minutes instead of 8 to 11 minutes, and staff can attend to other duties during this time. AOBP is the recommended approach to OBPM by Hypertension $\mathrm{Canada}^{7}$ and by the National Heart Foundation of Australia. ${ }^{6,33}$ The goal of our study was to effectively implement AOBP and HBPM measurement programs in a primary care clinic setting to detect white-coat BP elevation, reduce unnecessary antihypertensive therapy, and improve hypertension control without the use of dedicated research staff, space, or time. We also evaluated metrics critical to sustainability of the program, including estimates of staff time, patient/ provider satisfaction, and patient engagement in HBPM.

\section{Methods}

\section{Study Setting}

This was a pragmatic, prospective, observational study implementing a new AOBP and HBPM pro- gram in a university, hospital-based, primary care internal medicine clinic with approximately 15,000 patient visits per year in Salt Lake City, Utah. The study was conducted over 13 months from December, 2015 through December, 2016, and was reviewed and deemed exempt by the Institutional Review Board of the University of Utah Medical Center.

\section{Study Population}

Patients were eligible for the study if their clinic visit $\mathrm{BP}$ was elevated as defined below, and they met the following criteria: 1) no prior diagnosis of hypertension, or treated hypertension on a stable dose of antihypertensive medication; 2) considered suitable by their primary care clinician for HBPM without known adherence issues, cognitive impairments, severe depression, unstable cardiovascular disease, end-stage renal disease or dialysis treatment, or $\mathrm{BP} \geq 200 / 120 \mathrm{~mm} \mathrm{Hg} ; 3$ ) age $\geq 18$ years, not pregnant or lactating, adequate verbal or written English fluency; and 4) agreed to baseline HBPM education and to follow the recommended HBPM protocol. Patients with elevated clinic BP not enrolled during the 13 months of the study did not meet the entry criteria listed above, declined participation in the HBPM program, or were not approached for participation due to time constraints in the clinic. The nonenrolled patients were characterized for comparison with enrolled patients.

\section{Study Design}

The intended and finally implemented study designs are summarized in Figure 1. All BP measurements in the study were performed by clinic medical assistants (MAs) as described below in the BP measurement sections. By design, all clinic patients were to have a single, guideline-quality, observed, automated BP measurement performed by the MA. Those with $\mathrm{BP} \geq 140 / 90 \mathrm{~mm} \mathrm{Hg}$ were then to undergo AOBP with 3 sequential automated BP measurements performed with the patient alone in the examination room. Those with AOBP $\geq 135 / 85$ $\mathrm{mm} \mathrm{Hg}^{7}$ were to be asked to consider participation in the HBPM program. However, in the early months of the study, this protocol was implemented by some, but not all the clinic MAs. As a result, 53 and 40 patients respectively, were mistakenly enrolled onto 
Figure 1. Flow chart for home blood pressure (BP) implementation. HTN, hypertension; AOBP, Automated office blood pressure; HBPM, home blood pressure measurement.

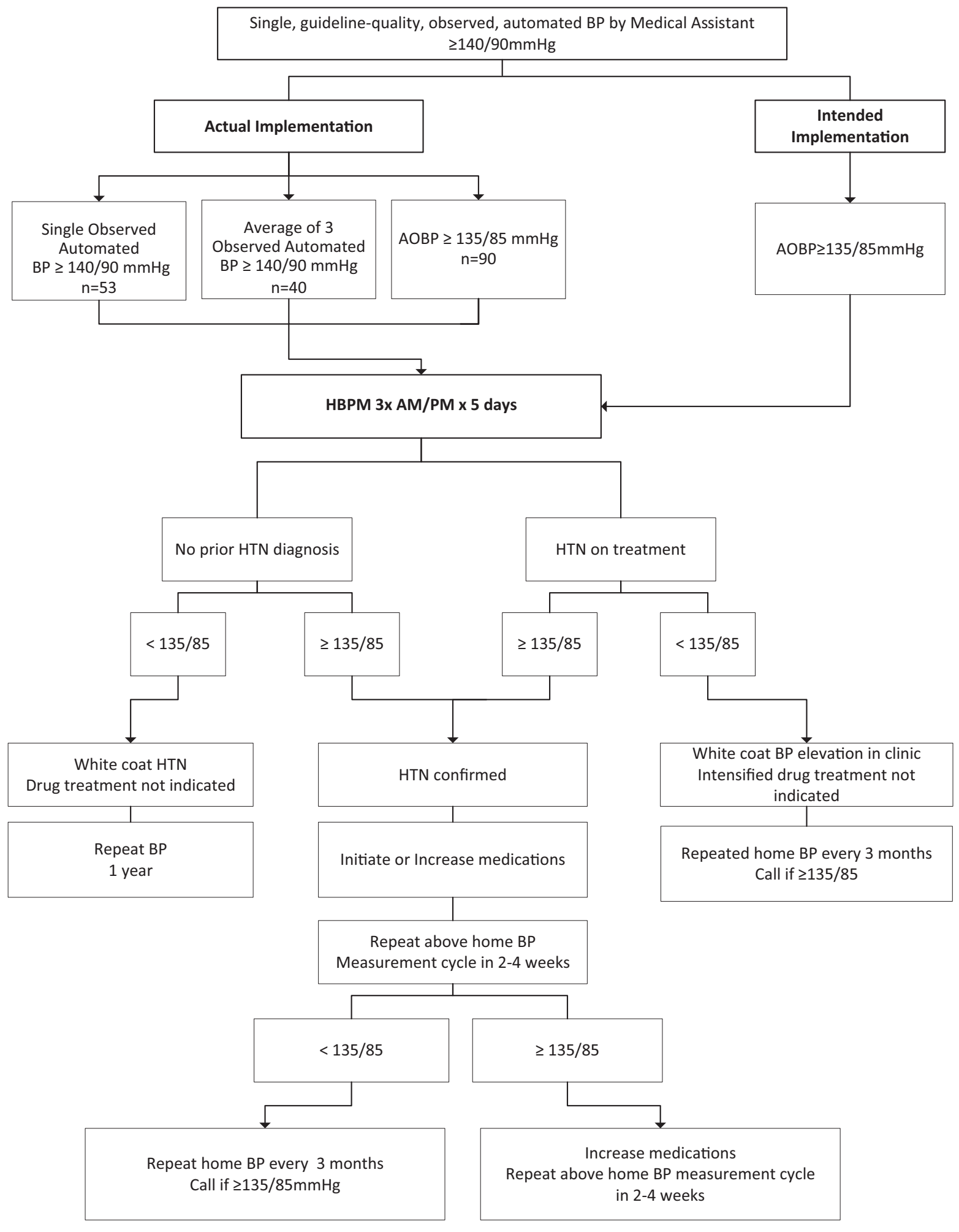

the HBPM program based on either a single, observed, automated BP measurement $\geq 140 / 90 \mathrm{~mm}$ $\mathrm{Hg}$ or based on the average of 3 observed automated

BP measurements $\geq 140 / 90 \mathrm{~mm} \mathrm{Hg}$. Ninety subjects were enrolled during the course of the study based on $\mathrm{AOBP} \geq 135 / 85 \mathrm{~mm} \mathrm{Hg}$. 
Based on initial HBPM results and hypertension status (prior or no prior hypertension diagnosis) patients were categorized into 4 groups (Figure 1). Patients not currently receiving antihypertensive medications whose average home $\mathrm{BP}$ was $\geq 135 / 85$ $\mathrm{mm} \mathrm{Hg}$ were diagnosed with new hypertension, and those with average home $\mathrm{BP}<135 / 85 \mathrm{~mm} \mathrm{Hg}$ were diagnosed with white-coat hypertension. ${ }^{7,10,33}$ Patients currently receiving antihypertensive medication were diagnosed with uncontrolled hypertension if average home $\mathrm{BP}$ was $\geq 135 / 85$ $\mathrm{mm} \mathrm{Hg}$ and with white-coat BP elevation if $<135 / 85 \mathrm{~mm} \mathrm{Hg}$.

Patients diagnosed with new hypertension or uncontrolled hypertension based on home BP measurement had antihypertensive medications initiated/ adjusted every 2 to 4 weeks by the clinic pharmacist during the first 9 months of the study and by the primary care physician during the last 4 months of the study (after departure of the clinic pharmacist) until home BP was controlled below 135/85 $\mathrm{mm} \mathrm{Hg}$.

\section{Office BP Measurement}

Clinic MAs and clinician staff were trained in a dedicated didactic session to perform guidelinequality office BP measurement according to current guidelines for clinical care ${ }^{34}$ and hypertension research studies. ${ }^{35}$ All office BP measurements were performed using a validated oscillometric upper arm automated device, the Omron HEM$907 \mathrm{XL}^{36}$ device that was used in the $\mathrm{ACCORD}^{37}$ and SPRINT ${ }^{38}$ hypertension trials, with an appropriate cuff size for midarm circumference. Before 1 to 3 observed OBPMs performed at 1-minute intervals, patients rested 5 minutes, seated in a chair with back supported, cuff at midsternal level, arm supported on a flat surface, feet flat on the floor, with no conversation during measurements. Correct measurement technique was monitored and reinforced during the study.

AOBP was performed with patients entirely alone in the examination room using the Omron HEM-907XL device set to average 3 sequential measurements at 1 minute intervals. According to current recommendations, a 5-minute rest period was not used before AOBP measurements. ${ }^{7}$ Otherwise, the techniques noted above were followed. An average AOBP $\geq 135 / 85 \mathrm{~mm} \mathrm{Hg}$ was used as an entry criteria to the study as this value corresponds to a daytime $\mathrm{ABPM} \geq 135 / 85 \mathrm{~mm} \mathrm{Hg}$. ${ }^{7,30,32}$

\section{Home BP Measurement}

Clinic staff were trained in recommended HBPM technique in a dedicated didactic session. ${ }^{39,40}$ Patient HBPM training included individual demonstration with observed confirmation of correct technique, provision of a written instruction summary sheet for home review, and an electronic link to an HBPM video to view in clinic or at home (Hypertension Canada: http://www.youtube.com/watch? $\mathrm{v}=$ eqadjdX5XU9Y7+=15s). Patients were instructed to take their BP twice daily after resting quietly in a chair for 5 minutes, legs uncrossed and feet flat on the floor, when under no subjective stress, before breakfast and before supper or at bedtime but before any $\mathrm{BP}$ medication intake. They were asked to collect 2 or $3 \mathrm{BP}$ measurements at 1-minute intervals, for 5 consecutive days, and then report the average of the 20 readings to the clinic pharmacist or medical staff via telephone, electronically through the electronic health record (EHR) secure messaging system, or in-person visits. ${ }^{41,42}$ Proper HBPM technique was reinforced during subsequent office visits and phone calls.

Eighty-four enrolled patients were loaned a validated HBPM device, the Omron $\mathrm{BP} 786 \mathrm{~N}^{43}$, which contains Bluetooth technology, a cuff that automatically adjusts to arms 9 to 17 inches in circumference, and has the capability to automatically take and average $3 \mathrm{BP}$ measurements at 1-minute intervals. Ninety-nine enrolled patients were allowed to use their own HBPM devices given that the monitors had been validated by 1 or more international protocols (www.medaval.org). The accuracy of both loaned and patient-owned devices was confirmed against the office Omron HEM907XL according to prior recommendations. ${ }^{44}$

\section{Patient and Provider Questionnaires}

Questionnaires were designed by the study investigators to determine patient and staff satisfaction with the program, time spent on the program, and intentions to continue with the program in the future. Questionnaire items were determined by face validity, and satisfaction was measured on a 5 -point Likert scale (strongly agree-strongly disagree; Appendices 1 and 2). Strongly agree/agree and strongly disagree/disagree categories were collapsed when presenting results. Patient questionnaires were mailed to patients in 2 waves and returned anonymously; the second survey wave included a $\$ 5$ compensation for completion. Provider 
Table 1. Characteristics of Clinic Patients with Elevated Blood Pressure, Both Enrolled and Not Enrolled in BP Monitoring Program

\begin{tabular}{|c|c|c|}
\hline Patient Characteristic & Enrolled $(\mathrm{n}=183)$ & Not Enrolled $(\mathrm{n}=1326)$ \\
\hline Mean age, years (SD) & $59.7(15.8)$ & $61.0(15.9)$ \\
\hline Sex, $\mathrm{n},(\%$ male $)$ & $103(56)$ & $701(53)$ \\
\hline Race, n, (\% white) & $164(90)$ & $1145(86)$ \\
\hline Insurance type, (n \% commercial) & $96(52)$ & $620(47)$ \\
\hline \multicolumn{3}{|l|}{ Comorbid medical conditions } \\
\hline Diabetes, n (\%) & $36(20)$ & $326(25)$ \\
\hline Cardiovascular disease, $\mathrm{n}(\%)$ & $47(26)$ & $424(32)$ \\
\hline Hyperlipidemia, n (\%) & $95(52)$ & $757(57)$ \\
\hline Chronic kidney disease*, n (\%) & $17(9)$ & $160(12)$ \\
\hline Hypertension diagnosis in EHR, $\mathrm{n}(\%)$ & $119(65)$ & $919(69)$ \\
\hline Median number of BP medications (25th percentile, 75 th percentile) & $2.0(1.0,2.0)$ & $2.0(1.0,2.0)$ \\
\hline Median baseline SBP (25th percentile, 75 th percentile) & $145.0(138.0,154.0)$ & $144(140.0,152.0)$ \\
\hline Median baseline DBP (25th percentile, 75 th percentile) & $91.0(85.0,96.0)$ & $90.0(81.0,94.0)$ \\
\hline
\end{tabular}

*Patients with ESRD were excluded from BP monitoring program.

SD, standard deviation; BP, blood pressure; EHR, electronic health record; SBP, systolic blood pressure; DBP, diastolic blood pressure; ESRD, end stage renal disease.

questionnaires were distributed in clinic and returned anonymously. A separate anonymous questionnaire was mailed to patients to self-assess their adherence to the recommended techniques of HBPM (Appendix 3). In this questionnaire, we assessed domains of proper HBPM that corresponded to those in the HBPM instructions provided to patients.

\section{Time Estimates for Staff}

Clinic staff were asked estimate the average time per patient needed to perform project clinical tasks: unobserved AOBP measurement, patient training for HBPM, HBPM device accuracy assessment, EHR documentation of AOBP/HBPM data, and transmission of HBPM data to the clinician.

\section{Statistical Analysis}

Descriptive statistics were collected for patient characteristics, including baseline systolic and diastolic BP. Diagnoses determined after receipt of initial HBPM results were stratified by OBPM technique and a Fisher's exact test was used to determine whether the proportion of diagnoses differed by OBPM technique.

\section{Results}

\section{Characteristics of Enrolled Patients}

Characteristics of the 183 enrolled patients and 1326 nonenrolled patients with elevated BP seen in our clinic during the same time period are listed in Table 1 . The average age of patients enrolled was 59.7 years, of which $56 \%$ were males and $90 \%$ were white. Comorbid medical conditions included an existing diagnosis of hypertension (65\%), hyperlipidemia $(52 \%)$, cardiovascular disease $(26 \%)$, and diabetes (20\%). Patients with previously diagnosed hypertension used an average of 1.8 antihypertensive medications. Enrolled patients were similar to nonenrolled patients.

The average clinic BP of the 183 enrolled patients was 147.3/90.0 mm Hg. Average BPs were $150.6 / 95.0 \mathrm{~mm} \mathrm{Hg}$ for the average of 3 observed BP measurements subgroup, 149.1/87.8 mm Hg for unobserved AOBP subgroup, and 146.8/90.4 $\mathrm{mm} \mathrm{Hg}$ for the single observed BP measurement subgroup. Of note, although all enrolled patients were to have clinic AOBP measurement, only half did (Tables 2, A and B).

\section{Collection of Initial Home BP Data and Related Diagnoses}

Of 183 enrolled patients, 164 (90\%) reported their initial average home BP readings to the clinic: $61 \%$ by phone, $32 \%$ by electronic messaging to the $\mathrm{EHR}$, and $6 \%$ by an in-person clinic visit. Average initial home BP values were 131.9/86.5 $\mathrm{mm} \mathrm{Hg}$ for the average of 3 observed BP measurements subgroup, 133.1/81.9 $\mathrm{mm} \mathrm{Hg}$ for unobserved AOBP 
Table 2A. Diagnoses of Patients Not Initially on Medications after Initial HBPM Results Received, Stratified by office Blood Pressure Technique $(n=84)$

\begin{tabular}{lccc}
\hline & \multicolumn{3}{c}{ Method of OBPM } \\
\cline { 2 - 4 } Diagnosis & $\begin{array}{c}\text { AOBP } \geq 135 / 85 \\
(\mathrm{n}=42)\end{array}$ & $\begin{array}{c}\text { Observed Average of 3 } \\
\mathrm{BP} \geq 135 / 85(\mathrm{n}=19)\end{array}$ & $\begin{array}{c}\text { Single Observed } \\
\mathrm{BP} \geq 140 / 90(\mathrm{n}=23)\end{array}$ \\
\hline New HTN, n (\%) & $22(52)$ & $12(63)$ & $11(48)$ \\
White coat HTN, n (\%) & $14(33)$ & $4(21)$ & $11(48)$ \\
Diagnosis unknown (no initial HBPM received), $\mathrm{n}(\%)$ & $6(14)$ & $3(16)$ & $1(4)$ \\
\hline
\end{tabular}

Fisher's exact test was used to determine whether the proportion of diagnoses differed by OBPM technique $(P=.39)$.

AOBP, automated office blood pressure; BP, blood pressure; HBPM, home blood pressure measurement; OBPM, office blood pressure measurement; HTN, hypertension.

subgroup, and 129.9/82.9 $\mathrm{mm} \mathrm{Hg}$ for the single observed BP measurement subgroup.

Of patients with elevated office BP and not on medication at enrollment, 29 (35\%) were found to have white-coat hypertension with average home $\mathrm{BP}<135 / 85 \mathrm{~mm} \mathrm{Hg}$ (Table 2A). The prevalence of white-coat hypertension did not significantly differ according to the method of OBPM: $21 \%$ for the observed average of 3 measurements, $33 \%$ for unobserved AOBP, and $48 \%$ for single observed BP $(P=.39)$. Forty-five patients (54\%) were diagnosed with new hypertension by HBPM, while 10 patients $(12 \%)$ never provided HBPM results to the clinic.

Of patients on antihypertensive medication at enrollment, 37 (37\%) were found to have whitecoat BP elevation with average home $\mathrm{BP}<135 / 85$ $\mathrm{mm} \mathrm{Hg}$ (Table 2B). Again, the frequency of whitecoat BP elevation did not differ according to the method of OBPM: white-coat BP elevation was diagnosed in $24 \%$ of patients with an observed average of $3 \mathrm{BP}$ measurements, $42 \%$ of patients with unobserved AOBP, and $40 \%$ of patients with a single observed BP measurement $(P=.19)$. Fifty- three patients (54\%) were diagnosed with uncontrolled hypertension, while 9 patients $(9 \%)$ did not provide HBPM results to the clinic.

\section{Rates of Home BP Control in Patients with New Diagnosis of Hypertension or Uncontrolled Hypertension}

During the course of the 13 month study, 37 of 45 (82\%) patients with a new diagnosis of hypertension provided additional serial HBPM results to facilitate initiation and adjustment of antihypertensive medications. Nineteen patients $(51 \%)$ achieved average home $\mathrm{BP}<135 / 85 \mathrm{~mm} \mathrm{Hg}$. Thirty-nine of 53 patients $(74 \%)$ with uncontrolled hypertension provided serial HBPM results, and 18 of the 39 patients (46\%) achieved average home $\mathrm{BP}<135 / 85$ $\mathrm{mm} \mathrm{Hg}$. We were not able to obtain serial HBPM measurements in 22 of 98 hypertensive patients (22\%) despite repeated attempts to contact them.

\section{Provider/Staff Satisfaction Questionnaires and Time Estimates}

Nineteen staff members received and completed the anonymous questionnaire (12 physicians in-

Table 2B. Diagnoses of Patients Initially on Medications after Initial and Final HBPM Results Received, Stratified by Office Blood Pressure Technique $(n=99)$

\begin{tabular}{|c|c|c|c|}
\hline \multirow[b]{2}{*}{ Diagnosis } & \multicolumn{3}{|c|}{ Method of OBPM } \\
\hline & $\begin{array}{c}\mathrm{AOBP} \geq 135 / 85 \\
(\mathrm{n}=48)\end{array}$ & $\begin{array}{l}\text { Observed Average of } 3 \mathrm{BP} \geq 135 / 85 \\
\qquad(\mathrm{n}=21)\end{array}$ & $\begin{array}{c}\text { Single Observed } \mathrm{BP} \geq 140 / 90 \\
(\mathrm{n}=30)\end{array}$ \\
\hline Existing uncontrolled HTN, n (\%) & $21(44)$ & $15(71)$ & $17(57)$ \\
\hline White-coat BP elevation, n (\%) & $20(42)$ & $5(24)$ & $12(40)$ \\
\hline $\begin{array}{l}\text { Unknown (no initial HBPM received), } \\
\text { n (\%) }\end{array}$ & $7(14)$ & $1(5)$ & $1(3)$ \\
\hline
\end{tabular}

Fisher's exact test was used to determine whether the proportion of diagnoses differed by OBPM technique $(P=.19)$. AOBP, automated office blood pressure; BP, blood pressure; HBPM, home blood pressure measurement; OBPM, office blood pressure measurement; HTN, hypertension. 
Table 3. AOBP and HBPM Time Requirements to Clinic and Medical Assistants

\begin{tabular}{lc}
\hline & $\begin{array}{c}\text { Estimated Time } \\
\text { Required (minutes) }\end{array}$ \\
\hline Unobserved* AOBP measurement & 4 \\
Patient training for HBPM & 10 \\
HBPM device accuracy assessment & 10 \\
EHR documentation of AOBP/HBPM & 2 \\
data & 2 \\
Transmission of HBPM data to clinician & 2 \\
\hline
\end{tabular}

*MAs could attend to other duties at this time.

AOBP, automated office blood pressure measurement; EHR, electronic health record; HBPM, home blood pressure measurement.

cluding 10 resident physicians, 5 MAs, and 2 pharmacists). Only a small minority (16\%) agreed that the AOBP/HBPM program required excessive time/effort; 68\% disagreed (Supplemental Table 1). Eighty-four percent of clinic staff respondents indicated they were adequately trained to effectively educate patients in proper HBPM techniques, and $89 \%$ estimated spending 1 to 15 minutes per patient on the project. Notably, 2 respondents estimated spending 31 to 45 minutes per patient on the project. The MA estimates of the specific time requirements for various tasks in the AOBP/HBPM program are listed in Table 3 . Overall, $68 \%$ of clinic staff agreed that the program positively influenced hypertension management in the clinic.

\section{Patient Satisfaction and HBPM Technique Questionnaires}

The patient satisfaction questionnaire had a $45 \%$ response rate. Ninety-one percent of respondents indicated that the instructions for HBPM were "easy to follow" and 63\% spent fewer than 15 minutes per day measuring $\mathrm{BP}$ on $\mathrm{BP}$ measurement days (Supplemental Table 2). Over $80 \%$ of respondents agreed that the project made them more involved in the care of their hypertension and improved their confidence to accurately measure their BP. Still, $26 \%$ of respondents did not plan to continue home $\mathrm{BP}$ monitoring after project completion.

The patient technique questionnaire had a $43 \%$ response rate. Despite prior intensive HBPM education, a substantial number of patients continued to use incorrect BP measurement technique that could invalidate their HBPM results. Overall, only a small minority $(13 \%)$ of respondents indicated proper/desired technique in all domains. Errors were most common in incorrect/inadequate rest period before measurement (52\%); failure to send results to clinic (33\%); and failure to refrain from other activity during measurement (29\%).

\section{Discussion}

This initiative demonstrates that an AOBP/HBPM program can be effectively implemented in a nonresearch primary care setting to detect white-coat BP elevation and reduce unnecessary antihypertensive therapy with favorable patient/provider satisfaction and patient engagement in HBPM. However, there are some important barriers to implementation that need to be overcome to maximize program success.

About one third of our patients with elevated clinic BP had white-coat hypertension in untreated patients or white-coat BP elevation in previously treated patients, similar to the $15 \%$ to $30 \%$ prevalence of white-coat hypertension in untreated patients and $25 \%$ to $38 \%$ prevalence of white-coat BP elevation in treated patients found in prior studies. ${ }^{4,5,45}$ AOBP has been demonstrated in multiple studies to reduce white-coat $\mathrm{BP}$ elevation and the subsequent need for out-of-office BP measurement $\mathrm{t}^{30-32}$ but we could not confirm its utility in our study. While initial average home BP was somewhat higher in the unobserved AOBP subgroup than in the 2 observed BP measurement subgroups (133.1 $\mathrm{mm} \mathrm{Hg}$ vs $131.9 \mathrm{~mm} \mathrm{Hg}$ and $129.9 \mathrm{~mm} \mathrm{Hg}$ ), there was no significant difference among the 3 subgroups in the prevalence of whitecoat BP elevation. This finding may have been related to our small sample size.

Initiation and/or intensification of antihypertensive medications in patients with elevated home BP who submitted subsequent HBPM results controlled home $\mathrm{BP}<135 / 85 \mathrm{~mm} \mathrm{Hg}$ in $51 \%$ and $46 \%$ of new and uncontrolled hypertensive patients, respectively. These control rates are similar to the 6-to-12-month hypertension control rates of $54 \%$ to $71 \%$ noted in 4 HBPM studies using telemonitoring with independent pharmacist comanagement ${ }^{18,19,46,47}$ and the 53\% 12-month control rate in a study without telemonitoring that used direct HBPM feedback to primary care clinicians. ${ }^{48}$

Primary care clinicians have expressed several concerns about implementing AOBP and HBPM 
in a nonresearch setting: whether an AOBP/HBPM program can be efficiently incorporated into daily clinical practice; whether patients will follow through with HBPM despite its substantial time/ effort commitment; and whether patients will perform HBPM with sufficient accuracy. ${ }^{14,49}$ With respect to the first concern about clinic efficiency, $68 \%$ of our clinic staff reported that the AOBP/ HBPM program positively affected hypertension management and did not require excessive time/ effort, an interpretation supported by the estimated time commitments noted in Table 3. While we did not objectively assess staff time commitments to the AOBP/HBPM program, 2 out of 19 respondents reported excessive time commitments of 31 to 45 minutes per patient. Perceived time burden may be more important than actual time commitment for program success. However, despite initial staff education about AOBP purpose and technique, MAs continued to perform observed BP measurements on some patients rather than unobserved AOBP early in the study. Effective educational materials along with frequent reinforcement and monitoring may be necessary to affect this culture-shift in OBPM.

Most of our enrolled patients followed through with $\mathrm{HBPM}$ as $90 \%$ provided requested initial HBPM results, and $74 \%$ of questionnaire respondents indicated they would continue HBPM in the future. Still, even with provision of a free loaned HBPM monitor and frequent telephone followup, $10 \%$ of enrolled patients provided no initial HBPM results, $22 \%$ provided no subsequent HBPM results despite their elevated home BP, and $26 \%$ of questionnaire respondents did not plan to continue using HBPM. Prior HBPM studies note that up to $20 \%$ of patients decline to initiate $\mathrm{HBPM}^{18}$ and report rates of submission of HBPM results of 70\% to $91 \%$ with dedicated pharmacist support ${ }^{46,47}$ but as low as $32 \%$ to $36 \%$ when such support was unavailable. ${ }^{27,48}$ Studies are needed to determine which patients are more likely not to complete HBPM and whether directed intensive patient education and shared decision making can minimize this barrier.

Potentially inaccurate HBPM results concern primary care clinicians and contribute to clinician therapeutic inertia to intensify antihypertensive therapy. ${ }^{14,50}$ Patient technique questionnaire results indicate continued patient errors in HBPM technique despite intensive education that could reduce $\mathrm{HBPM}$ accuracy and therefore undermine primary care clinician confidence in the HBPM intervention. Our "perfect adherence" results are similar to or even lower than those of previous studies that found only $18 \%$ to $53 \%$ of patients were sufficiently adherent to HBPM procedures to assure reasonable accuracy. ${ }^{26,27}$ Active patient education interventions and frequent reinforcement may be necessary to assure quality HBPM measurement, and we identified areas on which additional patient education could be focused: rest time, transmission of results, and avoiding other activities during measurement. ${ }^{51-53}$

Study strengths include a representative adult primary care population in a real-world clinic setting without use of dedicated research staff, time, or space. Telemonitoring was not used, and patients did not require computer access to report HBPM results. Observed office BP and AOBP were performed using a validated device according to current guideline recommendations ${ }^{7,34} \mathrm{HBPM}$ devices and BP measurement protocols adhered to current guidelines ${ }^{39-44}$, and HBPM training was intensive and frequently reinforced.

Study limitations include a single-center setting with limited minority population. Although we did use a clinical pharmacist, who had been previously assigned to the clinic for the first 9 months of the 13-month study, the primary care clinicians assumed all her duties for patient followup for the final 4 months. There are potential limitations to using HBPM rather than 24-hour ABPM to diagnose hypertension. HBPM is subject to inaccurate patient measurement technique and underreporting of high HBPM results. We could not monitor for underreporting as most patients reported HBPM results by telephone or the EHR. We did not use telemonitoring due to expense and did not require patients to bring in devices for download to minimize patient burden. HBPM may misclassify approximately $25 \%$ of true hypertension patients as having white-coat $\mathrm{BP}$ elevation if 24-hour ABPM is used as the standard diagnostic test ${ }^{54,55}$; however, it remains uncertain whether 24hour ABPM indeed provides superior prediction of cardiovascular risk compared with HBPM. ${ }^{56}$ While adjusting antihypertensive therapy according to HBPM results rather than office $\mathrm{BP}$ has not yet been proven by a randomized trial to provide superior reduction of cardiovascular events, HBPM 
does provide superior cardiovascular event prediction in treated hypertension patients. ${ }^{57}$

\section{Conclusion}

An AOBP/HBPM initiative in a nonresearch setting successfully detected white-coat BP elevation in one third of patients with elevated office BP and facilitated hypertension control in one half of patients with elevated home BP who provided follow-up data. AOBP did not clearly reduce whitecoat $\mathrm{BP}$ elevation in our population, and there were early-on challenges in having staff consistently perform AOBP.

The program was well accepted with respect to feasibility and efficiency by most clinic staff and patients. Barriers to program implementation included a temporary adjustment period for clinic staff to correctly use AOBP, about one fifth of patients failing to provide follow-up HBPM results, and many patients reporting incorrect HBPM technique despite intensive education. Future research should identify those patients most likely to perform and benefit from HBPM and determine how to best use shared decision making to optimize HBPM performance.

We express our gratitude to Dominick Ramirez, Shelbi Burgon, Kelsie Smith, Miriam Delgado, Jayci Bogue, Seth Wayman, Mariah Hennefer, Joyce Young, Medical Assistants, Iris Li, and our study participants.

To see this article online, please go to: http://jabfm.org/content/ 31/3/375.full.

\section{References}

1. Myers MG. The great myth of office blood pressure measurement. J Hypertens 2012;30:1894-8.

2. Myers MG, Godwin M, Dawes M, Kiss A, Tobe SW, Kaczorowski J. Measurement of blood pressure in the office: Recognizing the problem and proposing the solution. Hypertension 2010;55:195-200.

3. Powers BJ, Olsen MK, Smith VA, Woolson RF, Bosworth HB, Oddone EZ. Measuring blood pressure for decision making and quality reporting: Where and how many measures? Ann Intern Med 2011;154:781-8.

4. Gorostidi M, Vinyoles E, Banegas JR, de la Sierra A. Prevalence of white-coat and masked hypertension in national and international registries. Hypertens Res 2015;38:1-7.

5. Piper MA, Evans CV, Burda BU, Margolis KL, O'Connor E, Whitlock EP. Diagnostic and predictive accuracy of blood pressure screening methods with consideration of rescreening intervals: A sys- tematic review for the U.S. Preventive Services Task Force. Ann Intern Med 2015;162:192-204.

6. The National Heart Foundation of Australia. Guideline for the diagnosis and management of hypertension in adults-2016. Melbourne, Australia: The National Heart Foundation of Australia; 2016.

7. Leung AA, Daskalopoulou SS, Dasgupta K, et al. Hypertension Canada's 2017 guidelines for diagnosis, risk assessment, prevention, and treatment of hypertension in adults. Can J Cardiol 2017;33:557-76.

8. Whelton PK, Carey RM, Aronow WS, et al. 2017 ACC/AHA/AAPA/ABC/ACPM/AGS/APhA/ASH/ ASPC/NMA/PCNA guideline for the prevention, detection, evaluation, and management of high blood pressure in adults: A report of the American College of Cardiology/American Heart Association task force on clinical practice guidelines. J Am Coll Cardiol 2017 [Epub ahead of print].

9. Blacher J, Halimi JM, Hanon O, et al. Management of hypertension in adults: The 2013 French society of hypertension guidelines. Fundam Clin Pharmacol 2014;28:1-9.

10. Krause T, Lovibond K, Caulfield M, McCormack T, Williams B, Group GD. Management of hypertension: Summary of NICE guidance. BMJ 2011;343: d4891.

11. Siu AL. Screening for high blood pressure in adults: U.S. Preventive Services Task Force recommendation statement. Ann Intern Med 2015;163:778-86.

12. Shimbo D, Kent ST, Diaz KM, et al. The use of ambulatory blood pressure monitoring among Medicare beneficiaries in 2007-2010. J Am Soc Hypertens 2014;8:891-7.

13. Woolsey S, Brown B, Ralls B, Friedrichs M, Stults B. Diagnosing hypertension in primary care clinics according to current guidelines. J Am Board Fam Med 2017;30:170-7.

14. Kronish IM, Kent S, Moise N, et al. Barriers to conducting ambulatory and home blood pressure monitoring during hypertension screening in the United States. J Am Soc Hypertens 2017;11:573-80.

15. Kent ST, Shimbo D, Huang L, et al. Rates, amounts, and determinants of ambulatory blood pressure monitoring claim reimbursements among Medicare beneficiaries. J Am Soc Hypertens 2014;8:898-908.

16. Ostchega Y, Zhang G, Kit BK, Nwankwo T. Factors associated with home blood pressure monitoring among US adults: National Health and Nutrition Examination Survey, 2011-2014. Am J Hypertens 2017;30:1126-32.

17. Tirabassi J, Fang J, Ayala C. Attitudes of primary care providers and recommendations of home blood pressure monitoring-DocStyles, 2010. J Clin Hypertens (Greenwich) 2013;15:224-9.

18. Milani RV, Lavie CJ, Bober RM, Milani AR, Ventura HO. Improving hypertension control and patient engagement using digital tools. Am J Med 2017;130:14-20. 
19. Margolis KL, Asche SE, Bergdall AR, et al. Effect of home blood pressure telemonitoring and pharmacist management on blood pressure control: A cluster randomized clinical trial. JAMA 2013;310:46-56.

20. McManus RJ, Mant J, Haque MS, et al. Effect of self-monitoring and medication self-titration on systolic blood pressure in hypertensive patients at high risk of cardiovascular disease: The TASMIN-SR randomized clinical trial. JAMA 2014;312:799-808.

21. Omboni S, Gazzola T, Carabelli G, Parati G. Clinical usefulness and cost effectiveness of home blood pressure telemonitoring: Meta-analysis of randomized controlled studies. J Hypertens 2013;31:45567; discussion 467-8.

22. Tucker KL, Sheppard JP, Stevens R, et al. Selfmonitoring of blood pressure in hypertension: A systematic review and individual patient data metaanalysis. PLoS Med 2017;14:e1002389.

23. Ringrose JS, Polley G, McLean D, Thompson A, Morales F, Padwal R. An assessment of the accuracy of home blood pressure monitors when used in device owners. Am J Hypertens 2017;30:683-9.

24. Ruzicka M, Akbari A, Bruketa E, Kayibanda JF, Baril C, Hiremath S. How accurate are home blood pressure devices in use? A cross-sectional study. PLoS One 2016;11:e0155677.

25. Levy J, Gerber LM, Wu X, Mann SJ. Nonadherence to recommended guidelines for blood pressure measurement. J Clin Hypertens (Greenwich) 2016;18: $1157-61$.

26. Flacco ME, Manzoli L, Bucci M, et al. Uneven accuracy of home blood pressure measurement: A multicentric survey. J Clin Hypertens (Greenwich) 2015;17:638-43.

27. Milot JP, Birnbaum L, Larochelle P, et al. Unreliability of home blood pressure measurement and the effect of a patient-oriented intervention. Can J Cardiol 2015;31:658-63.

28. Logan AG, Dunai A, McIsaac WJ, Irvine MJ, Tisler A. Attitudes of primary care physicians and their patients about home blood pressure monitoring in Ontario. J Hypertens 2008;26:446-52.

29. Boivin JM, Tsou-Gaillet TJ, Fay R, Dobre D, Rossignol $\mathrm{P}$, Zannad $\mathrm{F}$. Influence of the recommendations on the implementation of home blood pressure measurement by French general practitioners: A 2004-2009 longitudinal survey. J Hypertens 2011; 29:2105-15.

30. Myers MG. Automated office blood pressure-The preferred method for recording blood pressure. J Am Soc Hypertens 2016;10:194-6.

31. Jegatheswaran J, Ruzicka M, Hiremath S, Edwards C. Are automated blood pressure monitors comparable to ambulatory blood pressure monitors? A systematic review and meta-analysis. Can J Cardiol 2017;33:644-52.
32. Myers MG. A short history of automated office blood pressure-15 years to SPRINT. J Clin Hypertens (Greenwich) 2016;18:721-4.

33. Gabb GM, Mangoni AA, Anderson CS, et al. Guideline for the diagnosis and management of hypertension in adults-2016. Med J Aust 2016;205:85-9.

34. Pickering TG, Hall JE, Appel LJ, et al. Recommendations for blood pressure measurement in humans and experimental animals: Part 1: blood pressure measurement in humans: A statement for professionals from the Subcommittee of Professional and Public Education of the American Heart Association Council on High Blood Pressure Research. Hypertension 2005;45:142-61.

35. TRUE Consortium. Recommended standards for assessing blood pressure in human research where blood pressure or hypertension is a major focus. Kidney Int Rep 2017;2:733-8.

36. White WB, Anwar YA. Evaluation of the overall efficacy of the Omron office digital blood pressure HEM-907 monitor in adults. Blood Press Monit 2001;6:107-10.

37. Cushman WC, Evans GW, Byington RP, et al. Effects of intensive blood-pressure control in type 2 diabetes mellitus. N Engl J Med 2010;362:1575-85.

38. SPRINT Research Group. A randomized trial of intensive versus standard blood-pressure control. N Engl J Med 2015;2015:2103-16.

39. Pickering TG, Miller NH, Ogedegbe G, Krakoff LR, Artinian NT, Goff D. Call to action on use and reimbursement for home blood pressure monitoring: A joint scientific statement from the American Heart Association, American Society Of Hypertension, and Preventive Cardiovascular Nurses Association. Hypertension 2008;52:10-29.

40. Sharman JE, Howes FS, Head GA, et al. Home blood pressure monitoring: Australian expert consensus statement. J Hypertens 2015;33:1721-8.

41. Niiranen TJ, Asayama K, Thijs L, et al. Optimal number of days for home blood pressure measurement. Am J Hypertens 2014;28:595-603.

42. Niiranen TJ, Johansson JK, Reunanen A, Jula AM. Optimal schedule for home blood pressure measurement based on prognostic data: The Finn-Home Study. Hypertension 2011;57:1081-6.

43. Takahashi H, Yokoi T, Yoshika M. Validation of the OMRON M6 Comfort (HEM-7321-E) upper arm blood pressure monitor, in oscillometry mode, for clinic use and self measurement in a general population, according to the European Society of Hypertension International Protocol revision. 2010. [Internet]. Dublin, UK: dablEducational Trust; 2014 Jan 22, p. 4.

44. Imai Y, Otsuka K, Kawano Y, et al. Japanese society of hypertension (JSH) guidelines for self-monitoring of blood pressure at home. Hypertens Res 2003;26: 771-82. 
45. Franklin SS, Thijs L, Hansen TW, O'Brien E, Staessen JA. White-coat hypertension: New insights from recent studies. Hypertension 2013;62:982-7.

46. Green BB, Cook AJ, Ralston JD, et al. Effectiveness of home blood pressure monitoring, Web communication, and pharmacist care on hypertension control: A randomized controlled trial. JAMA 2008;299: 2857-67.

47. Magid DJ, Olson KL, Billups SJ, Wagner NM, Lyons EE, Kroner BA. A pharmacist-led, American Heart Association Heart360 Web-enabled home blood pressure monitoring program. Circ Cardiovasc Qual Outcomes 2013;6:157-63.

48. DeAlleaume L, Parnes B, Zittleman L, et al. Success in the Achieving CARdiovascular Excellence in Colorado (A CARE) home blood pressure monitoring program: A report from the Shared Networks of Colorado Ambulatory Practices and Partners (SNOCAP). J Am Board Fam Med 2015;28:548-55.

49. Fletcher BR, Hinton L, Hartmann-Boyce J, Roberts NW, Bobrovitz N, McManus RJ. Self-monitoring blood pressure in hypertension, patient and provider perspectives: A systematic review and thematic synthesis. Patient Educ Couns 2016;99:210-9.

50. Kerr EA, Zikmund-Fisher BJ, Klamerus ML, Subramanian $\mathrm{U}$, Hogan MM, Hofer TP. The role of clinical uncertainty in treatment decisions for diabetic patients with uncontrolled blood pressure. Ann Intern Med 2008;148:717-727.

51. American Medical Association/Johns Hopkins Medicine. Self-measured blood pressure monitoring program: Engaging patients in self-measurement. Available from: www.stepsforward.org/Static/images/ modules/8/downloadable/SMBP\%20monitoring \%20program.pdf.

52. British Hypertension Society. Home blood pressure monitoring. 2014. Available from: http://bhsoc.org/ resources/hbpm.

53. Sharman JE, Howes F, Head GA, et al. How to measure home blood pressure: Recommendations for healthcare professionals and patients. Aust Fam Physician 2016;45:31-4.

54. Nunan D, Thompson M, Heneghan CJ, Perera R, McManus RJ, Ward A. Accuracy of self-monitored blood pressure for diagnosing hypertension in primary care. J Hypertens 2015;33:755-62; discussion 762.

55. Zhang L, Li Y, Wei FF, et al. Strategies for classifying patients based on office, home, and ambulatory blood pressure measurement. Hypertension 2015; 65:1258-65.

56. Shimbo D, Abdalla M, Falzon L, Townsend RR, Muntner P. Studies comparing ambulatory blood pressure and home blood pressure on cardiovascular disease and mortality outcomes: A systematic review. J Am Soc Hypertens 2016;10:224-34, e217.

57. Shimada K, Kario K, Kushiro T, et al. Prognostic significance of on-treatment home and clinic blood pressure for predicting cardiovascular events in hypertensive patients in the HONEST study. J Hypertens 2016;34:1520-7.

\section{Appendix 1}

\section{Patient Satisfaction Questionnaire: Home Blood Pressure Monitoring Project 2015 to 2016}

Please complete this short questionnaire regarding your participation in the Clinic 2 General Internal Medicine home blood pressure monitoring project. The goal of this questionnaire is to obtain your opinion on how to improve home blood pressure monitoring for patients. This questionnaire should take no longer than 10 minutes to complete. All responses will remain anonymous. Thank you for your time in completing this questionnaire.

Please check the one most appropriate answer for question 1 to 10 below:

\section{Question 1:}

My participation in the Clinic 2 home blood pressure monitoring project has improved my actual blood pressure numbers.

() Strongly agree

() Agree

() Neither agree nor disagree

() Disagree

() Strongly disagree

\section{Question 2:}

The home blood pressure monitoring project helped me become more involved in my care regarding my blood pressure.

() Strongly agree

() Agree

() Neither agree nor disagree

() Disagree

() Strongly disagree

\section{Question 3:}

I now have more confidence in correctly measuring my blood pressure at home.
() Strongly agree
() Agree
() Neither agree nor disagree
() Disagree
() Strongly disagree

\section{Question 4:}

I communicated my blood pressure numbers to my doctor or Clinic 2 staff by: 
Check all that apply

() Fax

() Mail

() MyChart electronic message

() Telephone

() Other (please write method here):

\section{Question 5:}

When I sent or called my home blood pressure measurements to Clinic 2, the clinic staff replied in a timely manner regarding any need for medication changes.

() Strongly agree

() Agree

() Neither agree nor disagree

() Disagree

() Strongly disagree

\section{Question 6:}

It was easy to follow the instructions to measure my blood pressure at home correctly.

() Strongly agree

() Agree

() Neither agree nor disagree

() Disagree

() Strongly disagree

\section{Question 7:}

How much time did you spend per day measuring your blood pressure at home during your participation in the blood pressure monitoring project?

() Less than 15 minutes

() 16 to 30 minutes

() 31 to 60 minutes

() More than 60 minutes

\section{Question 8:}

My overall impression of the home blood pressure monitoring project is:

() Very positive

() Positive

() Neutral

() Negative

() Very negative

\section{Question 9:}

If my clinic doctor had not been able to loan me a home blood pressure monitor (cuff), I would have been willing to spend this much to buy my own home blood pressure monitor (cuff):
() $\$ 1$ to $\$ 25$

() $\$ 26$ to $\$ 50$

() $\$ 51$ to $\$ 75$

() $\$ 76$ to $\$ 100$

() I already owned a blood pressure monitor during my participation in the project

() I would not have been willing to buy my own monitor (please specify if not affordable or other reason here):

\section{Question 10:}

I plan to continue to measure my blood pressure at home in the future:

() Yes

() No

Please continue on to the back page:

How can the Clinic 2 Internal Medicine staff improve home blood pressure monitoring for you?

Please write in any other comments about/approximately home blood pressure monitoring you would like to share with the Clinic 2 staff here:

\section{Appendix 2}

Staff Satisfaction Questionnaire: Clinic 2 General Internal Medicine 2015 to 2016 Home Blood Pressure Monitoring Project

Please take some time to complete this short questionnaire regarding your participation in the patient home blood pressure monitoring project. The goal of this questionnaire is to obtain your opinion on how to improve home blood pressure monitoring for patients. This questionnaire should take no longer than 15 minutes to complete. All responses will remain anonymous. Thank you for your time in completing this questionnaire.

Please select the 1 most appropriate answer for questions 1 to 8 below:

\section{Question 1:}

I am a:
() Medical Assistant
() Nurse or Pharmacist
() Resident Physician
() Attending Physician
() Other (please describe role): 
Question 2:

The home blood pressure project lowered blood pressure levels and positively affected blood pressure management for my patients.

() Strongly agree

() Agree

() Neither agree nor disagree

() Disagree

() Strongly disagree

() I do not know

\section{Question 3:}

For my patients, the home blood pressure monitoring project improved patient engagement in their own self-management of blood pressure.

() Strongly agree

() Agree

() Neither agree nor disagree

() Disagree

() Strongly disagree

() I do not know

\section{Question 4:}

The home blood pressure monitoring project required excessive effort on my part.

() Strongly agree

() Agree

() Neither agree nor disagree

() Disagree

() Strongly disagree

(If you agree or strongly agree, please explain):

\section{Question 5:}

I feel that I have been adequately trained to provide appropriate instruction for proper home blood pressure monitoring technique to my patients.

() Strongly agree

() Agree

() Neither agree nor disagree
() Disagree

() Strongly disagree

(If you disagree or strongly disagree, please explain):

\section{Question 6:}

I plan to continue educating my patients on the proper technique for home blood pressure monitoring.

() Strongly agree

() Agree

() Neither agree nor disagree

() Disagree

() Strongly disagree

\section{Question 7:}

My overall impression of the Clinic 2 home blood pressure monitoring project as a way to efficiently improve blood pressure control for my patients is:

() Very positive

() Positive

() Neutral

() Negative

() Very negative

\section{Question 8:}

On average I spent this amount of minutes per patient for the blood pressure monitoring project (in clinic and outside of patient appointments):

() 1 to 15 minutes

() 16 to 30 minutes

() 31 to 45 minutes

() 46 to 60 minutes

() More than 60 minutes

How could home blood pressure monitoring for patients be improved?

Any additional comments are welcomed here: 
Supplemental Table 1. Staff Satisfaction Measures $(n=19)$

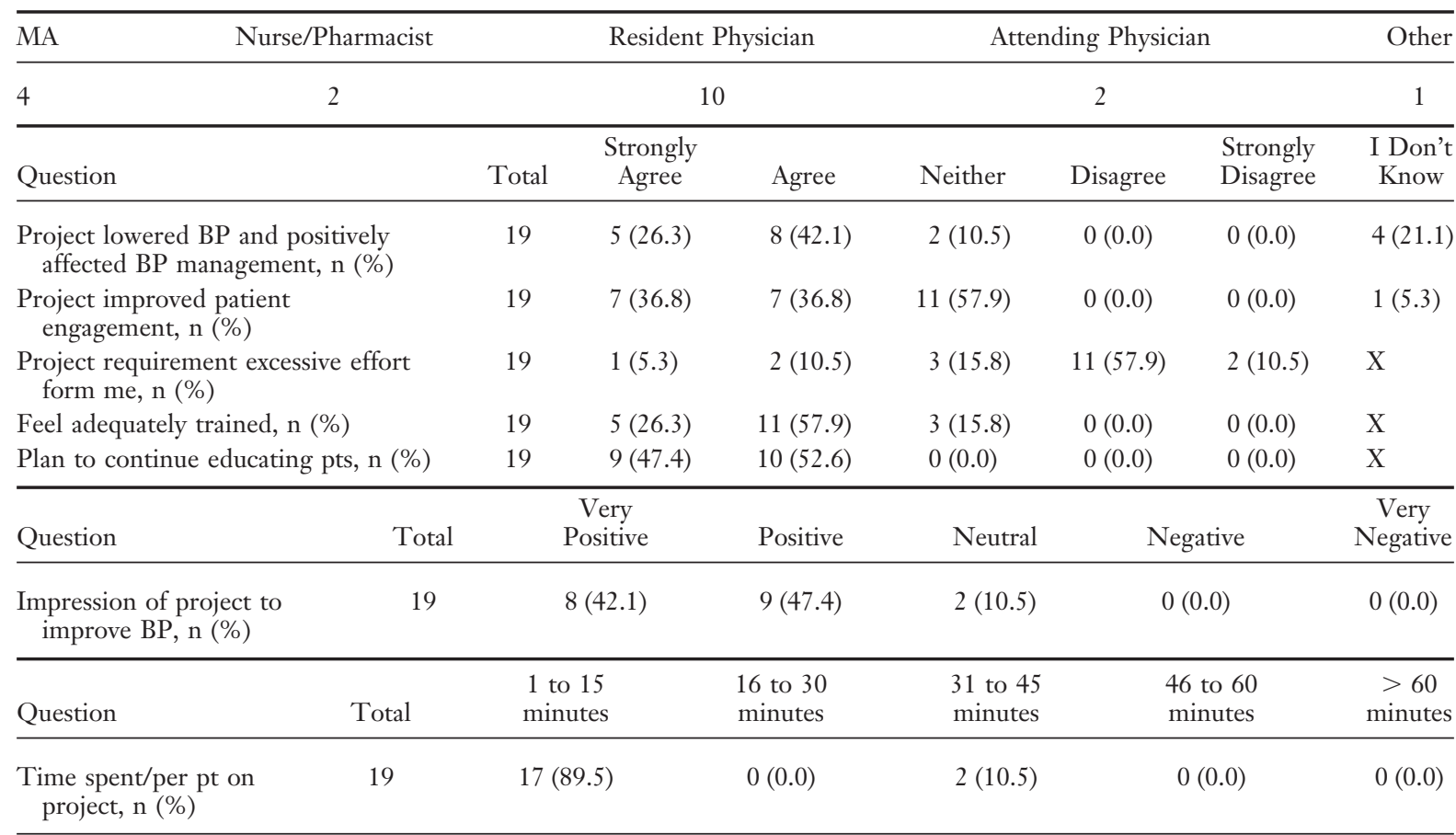

BP, blood pressure; MA, medical assistants.

Supplemental Table 2. Patient Satisfaction Measures $(n=55)$ Patient Satisfaction

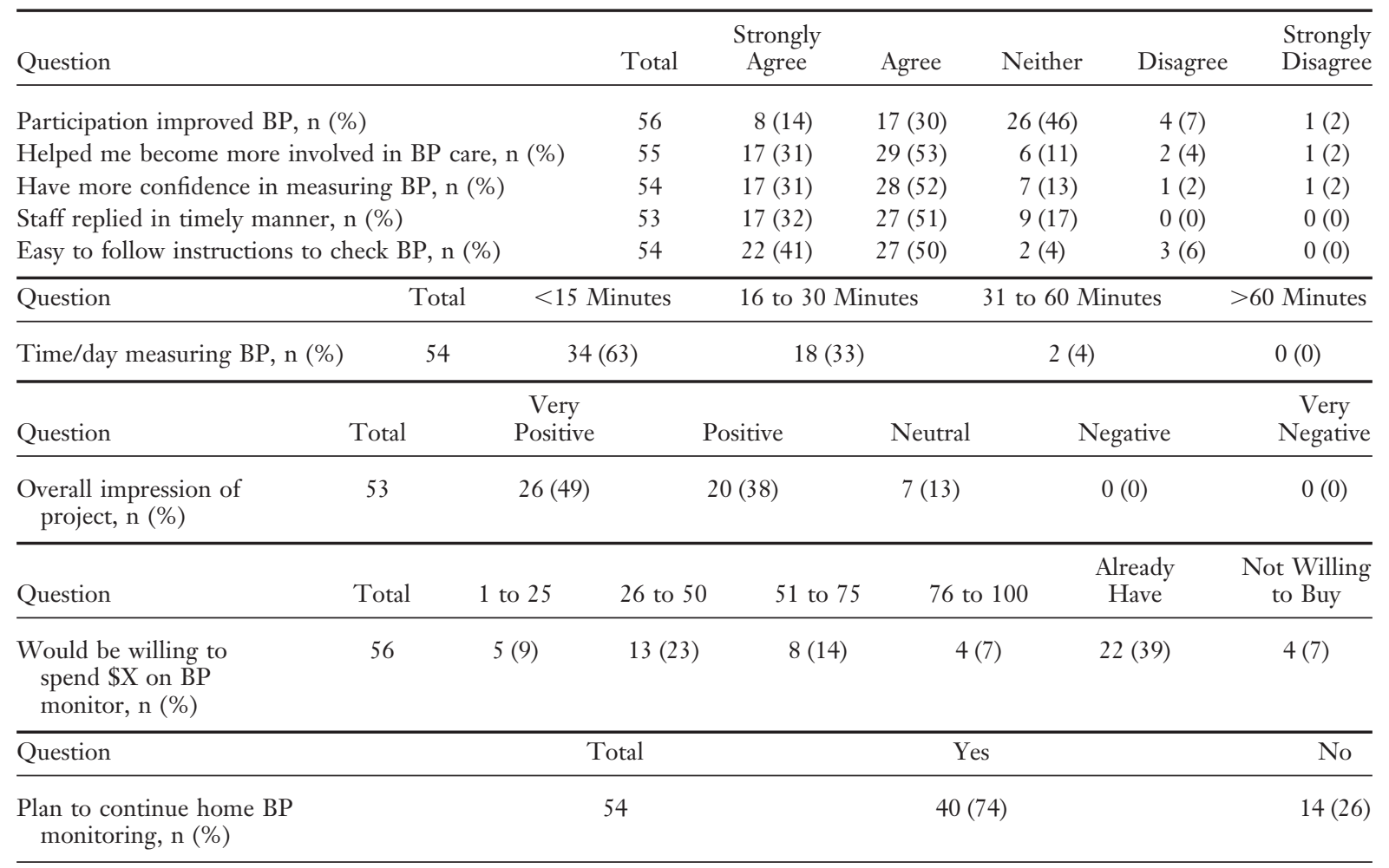

BP, blood pressure. 